\title{
Distributed UAV Deployment in Hostile Environment: A Game-theoretic Approach
}

\author{
Chen Han, Aijun Liu, Kang An, Gan Zheng, Fellow, IEEE, and Xinhai Tong
}

\begin{abstract}
As a new type of unmanned intelligent equipment, unmanned aerial vehicles (UAVs) have attracted growing attentions in various fields, such as electronic reconnaissance in jamming environments. However, the intense confrontation and high dynamics of the hostile jamming environments require a UAV swarm to achieve flexible and autonomous deployment optimization, and enhance anti-jamming capability. In this letter, a distributed UAV swarm's deployment approach is proposed where a congestion game is formulated to model the interaction among the UAV swarm. Particularly, each UAV independently adjusts the location, and realizes the efficient, flexible and dynamic position control, thus increasing the amount of collected data and enhancing the anti-jamming performance. Moreover, the Nash equilibrium of the formulated congestion game is analyzed, and simulation results verify the effectiveness of the proposed approach.
\end{abstract}

Index Terms-UAV swarm, Location deployment, Game theory, Distributed optimization

\section{INTRODUCTION}

As a new type of unmanned intelligent system, the unmanned aerial vehicle (UAV) swarm has been widely used in communication tasks such as electronic reconnaissance and data collection, due to its advantages of low cost and flexible deployment [1], [2]. As for the data collection task in hostile environments, the intense confrontation and high dynamics caused by the jammers have put forward high requirements for the deployment performance of UAVs [3].

There are various works in the existing literature on the deployment problem of UAVs. The authors in [4] studied deployment of UAVs by a differential evolution algorithm. In [5], the authors proposed a successive convex approximation based algorithm to optimize the UAVs' deployment. In [6], based on circle packing theory, the multi-UAV base station deployment was studied to provide wireless coverage to the ground users. The authors in [7] investigated the multi-UAV

This work was supported in part by the National Key Research and Development Program of China under Grant 2018YFB1801103, in part by the National Natural Science Foundation of China under Grant 61901516, 61901502, 62071352, in part by the Natural Science Foundation on Frontier Leading Technology Basic Research Project of Jiangsu Province under Grant BK20192002, in part by the National Postdoctoral Program for Innovative Talents under Grant BX20200101. (Corresponding author: Aijun Liu.)

Chen Han, Aijun Liu and Xinhai Tong are with the College of Communications Engineering, Army Engineering University, Nanjing 210007, China (e-mail: hanchen.lgd@gmail.com; liuaj.cn@163.com; tongxinhai2012@163.com).

Kang An is with the Sixty-Third Research Institute, National University of Defense Technology, Nanjing 21007, China (e-mail:ankang89@nudt.edu.cn)

Gan Zheng is with the Wolfson School of Mechanical, Electrical and Manufacturing Engineering, Loughborough University, Loughborough LE113TU, U.K. (e-mail: g.zheng@lboro.ac.uk).

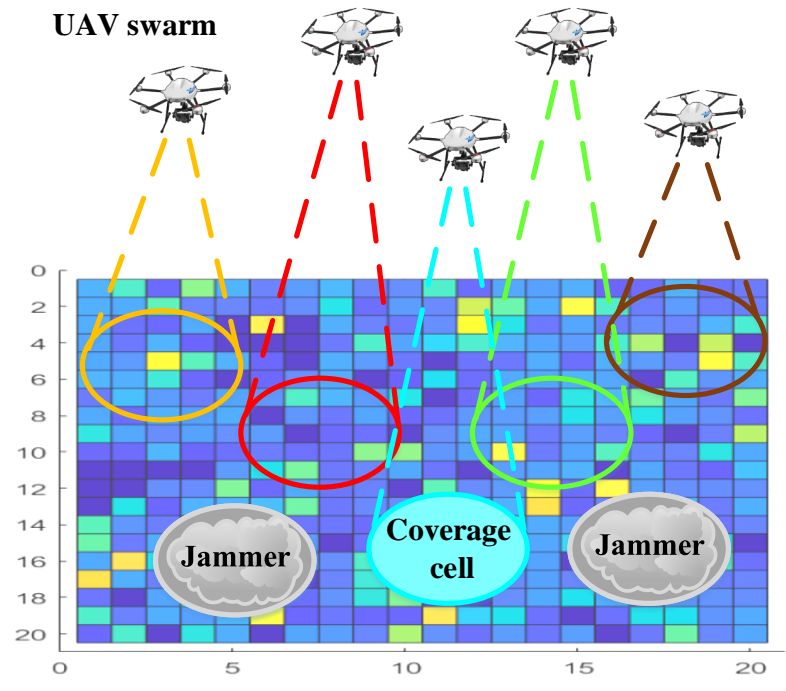

Fig. 1: System model. A UAV swarm carries data collection task over a meshed region, where there are hostile jammers randomly located in several grids.

deployment problem for data collection from ground internet of things (IoT) devices. In [8], the authors proposed a $\mathrm{K}$ means clustering-based UAV deployment approach to relay data for ground users in the UAVs-aided non-orthogonal multiple access (NOMA) system. The authors in [9] proposed heuristic algorithm to solve the deployment problem of UAVs which jointly considered UAVs' flying distances and service altitudes. In [10], the authors studied the optimal deployment of UAV networks with a high-resolution quantization theory.

However, in the hostile jamming environment, the above related works may face the following problems: i) these approaches mainly focus on the centralized optimization methods, which need certain prior and global information. However the complete and accurate global information is difficult to obtain in the hostile environment; ii) these works do not consider the high dynamics caused by jammers, which requires fast redeployment to cope with the rapidly changing jamming environment. Therefore, in this letter, a congestion game is formulated to model the interaction among the UAV swarm in the hostile jamming environment, and a distributed approach is proposed to obtain the sub-optimal deployment strategy, which is proved to be a stable Nash equilibrium solution.

\section{System Model And Problem Formulation}

As shown in Fig. 1, a UAV swarm, including $M$ UAVs, is used for collecting signals from the hostile environment. 
The target region is meshed into $X$ grids. The coordinate of each grid's center is used to represent this grid. The horizontal projection coordinate of the spatial position of UAV $u$ is $l_{u}$, which is the center of the grid as well. The sensing radius of each UAV is $L$, which means data generated within the sensing range can be collected. The amount of data that can be collected in the $v$-th grid, whose coordinate is $l_{v}=\left(x_{v}, y_{v}\right)$, follows the Poisson distribution, i.e., $T(v) \sim \mathcal{P}\left(\lambda_{v}\right)$ [11],

$$
\operatorname{Pr}\{T(v)=k\}=\frac{\left(\lambda_{v}\right)^{k}}{(k) !} e^{-\lambda_{v}},
$$

where $\lambda_{v}$ is the Poisson coefficient. If there is a ground-based jammer $J$ in $l_{v}$, then we set $\delta_{v}(J=1)=1$, i.e.,

$$
\delta_{v}(J=1)=\left\{\begin{array}{l}
1, \text { there is a jammer in } l_{v} \\
0, \text { there is no jammer in } l_{v}
\end{array} .\right.
$$

The Nakagami-m fading distribution is used to model the airground link, and the channel capacity of the UAV in the grid $l_{v}$ is:

$$
c(v)=B \log _{2}\left(1+\frac{h_{v}^{u} p_{U}}{\sigma^{2}+\delta_{v}(J=1) h_{J} p_{J}}\right),
$$

where $B$ and $\sigma^{2}$ are the channel bandwidth and channel noise. $p_{U}, p_{J}, h_{v}^{u}$, and $h_{J}$ are defined as the transmission power and channel gain of UAVs and jammers, respectively. The data can be collected in $l_{v}$ is given by:

$$
D(v)=\min \{T(v), c(v)\} .
$$

If $m(v)$ UAVs cover the same grid, they will collect the electromagnetic data repeatedly, then, as for the whole swarm, the actually available collected data is equivalent to $D(v) / m(v)$ for each UAV. However, to maximize each UAV's collecting capability, all UAVs share the same spectrum. Thus, the overlapping coverage of UAVs can lead to additional collection confusion because of the mutual interference, resulting in a further reduction in the available data. To address this issue, we introduce $\alpha, \alpha \geq 1$ to represent the impact caused by overlapped coverage among UAVs. Then, the amount of available data that each UAV can collect is defined as $D(v) / \alpha m(v)$ for simplification. Thus, the actual collection data of UAV $u$ is:

$$
\begin{aligned}
C(u) & =\sum_{v \in \Omega(u)} \frac{D(v)}{\alpha m(v)}, \\
\Omega(u) & =\left\{l_{v} \mid\left\|l_{u}-l_{v}\right\| \leq L\right\},
\end{aligned}
$$

where $\left\|l_{u}-l_{v}\right\|$ is the Euclidean distance from $l_{u}$ to $l_{v} . \Omega(u)$ is the grid set that UAV $u$ can collect data from. Then, the total data collected by the UAV swarm is:

$$
\mathbb{C}=\sum_{u=1}^{M} C(u)
$$

To perform data collection and transmission task in the hostile jamming environment, UAV swarm need to avoid jamming areas and move towards hot spots to collect more data efficiently. Meanwhile, the interference among UAVs within the swarm will also reduce the efficiency of data collection, and even lead to task failure. Additionally, the centralized optimization

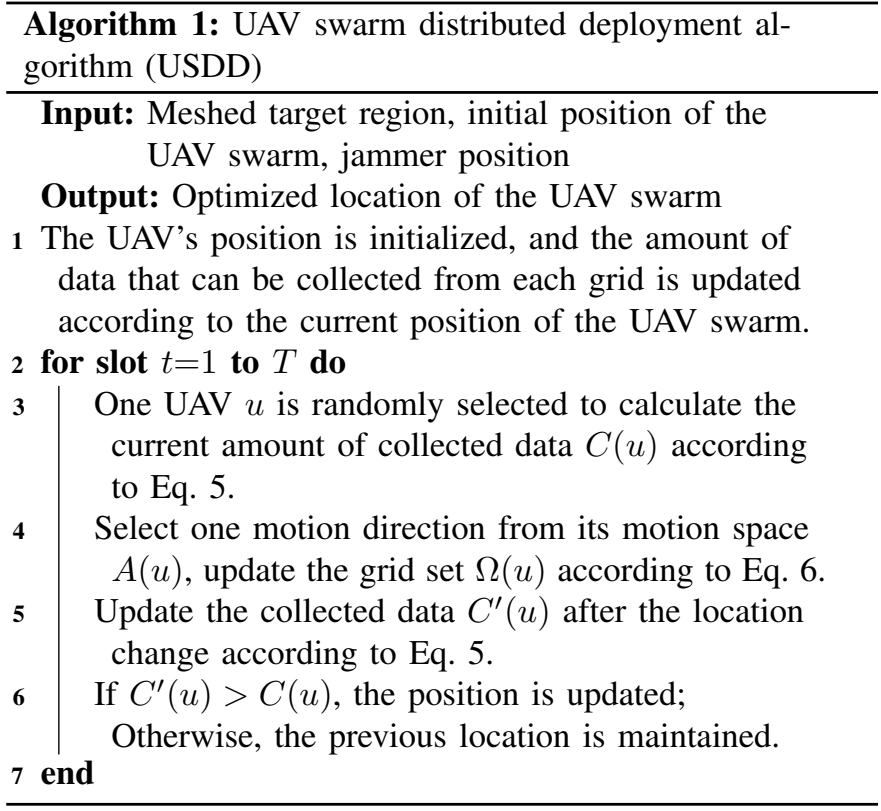

schemes often suffer from costly signaling overhead and larger delay, which is not conducive to real-time execution and flexibility for accomplishing the data collection task and against jamming attacks. Thus, our design objective is to maximize the collected data of the whole UAV swarm through distributed deployment, namely,

$$
P 1: \max _{\left\{l_{1}, l_{2}, \ldots l_{M}\right\}_{\text {uav }}} \mathbb{C} \text {. }
$$

In what follows, the entire UAV swarm is optimized in a distributed manner, with each UAV autonomously adjusting location to maximize collected data.

\section{UAV SWARM DISTRIBUTED DEPLOYMENT ALGORITHM}

\section{A. Algorithm description}

The distributed deployment problem of the UAV swarm can be modeled as a congestion game. Each UAV maximizes its own collected data through distributed optimization. The game utility function of each UAV is given by:

$$
r_{u}=C(u) .
$$

As shown in Algorithm 1, a UAV swarm's distributed deployment algorithm (USDD) is proposed, which utilizes the stochastic optimization. To begin with, a UAV $u$ along with its movement direction is randomly selected according to its motion space $A(u)=\left[a_{1}, a_{2}, a_{3}, a_{4}\right]$, i.e., Up, Down, Left and Right. Note that part of the action is unreachable if on the boundary. If the changed position can collect more data, then the UAV will follow that change; Otherwise it remains the previous location. With finite steps, this algorithm can obtain stable convergence results by iterative optimization, which can be proved in the Algorithm analysis.

\section{B. Algorithm analysis}

Theorem 1: The proposed congestion game is an exact potential game, and there is at least one stable Nash equilibrium point. 

[12].

Proof: First, the exact potential game is defined as follows

Definition 1: When the location of a UAV changes from $l_{u}$ to $l_{u^{\prime}}$, if there is a potential function $\Phi$ satisfying the following Eq. (10), the proposed game model is an exact potential game, and it has at least one pure strategy Nash equilibrium.

$$
r_{u}-r_{u^{\prime}}=\Phi\left(l_{u}, l_{-u}\right)-\Phi\left(l_{u^{\prime}}, l_{-u^{\prime}}\right) .
$$

where $l_{-u}$ represents the location of other UAVs except $u$, i.e., $(\{-u\}=\{[1, M] / u\})$.

Inspired by [13], a potential function is defined as:

$$
\Phi=\sum_{x \in[1, X]} \sum_{i=1}^{m(x)} \frac{D(x)}{\alpha i} .
$$

where $x \in[1, X]$ represents all the grids in the target area. When the location of a UAV is changed from $l_{u}$ to $l_{u^{\prime}}$, the utility increment of the UAV is:

$$
\begin{aligned}
r_{u}-r_{u^{\prime}} & =C(u)-C\left(u^{\prime}\right) \\
& =\sum_{i \in \Omega(u)} \frac{D(i)}{\alpha m(i)}-\sum_{j \in \Omega\left(u^{\prime}\right)} \frac{D(j)}{\alpha m^{\prime}(j)},
\end{aligned}
$$

where $\Omega(u)$ and $\Omega\left(u^{\prime}\right)$ respectively represent the grid set that can be collected data from when the UAV is located at $l_{u}$ and $l_{u}^{\prime}$. Limited by the movement modes of the UAV, i.e., the UAV can only move one grid at a slot, thus, there may be an intersection between $\Omega(u)$ and $\Omega\left(u^{\prime}\right)$. Define the intersection as $\Theta=\Omega(u) \cap \Omega\left(u^{\prime}\right)$. Then, for the grids in $\Theta$, the number of UAVs covering these grids does not change, and thus, the amount of data that each UAV can collect in these grids does not change as well, i.e.,

$$
\sum_{i \in \Theta} \frac{D(i)}{\alpha m(i)}=\sum_{j \in \Theta} \frac{D(j)}{\alpha m^{\prime}(j)} .
$$

Then, we can get,

$$
\begin{aligned}
r_{u}-r_{u^{\prime}} & =\sum_{i \in \Omega(u)} \frac{D(i)}{\alpha m(i)}-\sum_{j \in \Omega\left(u^{\prime}\right)} \frac{D(j)}{\alpha m^{\prime}(j)} \\
& =\sum_{i \in\{\Omega(u) / \Theta\}} \frac{D(i)}{\alpha m(i)}-\sum_{j \in\left\{\Omega\left(u^{\prime}\right) / \Theta\right\}} \frac{D(j)}{\alpha m^{\prime}(j)} .
\end{aligned}
$$

In this case, the set $\{\Omega(u) / \Theta\}$ is the grid set that was covered by UAV $u$ before the location change but is no longer covered now, i.e., $\forall k \in\{\Omega(u) / \Theta\}, m^{\prime}(k)=m(k)-1$. Similarly, $\forall k \in\left\{\Omega\left(u^{\prime}\right) / \Theta\right\}, m^{\prime}(k)=m(k)+1$.

When the location of the UAV is changed from $l_{u}$ to $l_{u^{\prime}}$, the increment of potential function is:

$$
\begin{aligned}
& \Phi\left(l_{u}, l_{-u}\right)-\Phi\left(l_{u^{\prime}}, l_{-u^{\prime}}\right) \\
& =\sum_{x \in[1, X]} \sum_{i=1}^{m(x)} \frac{D(x)}{\alpha i}-\sum_{y \in[1, X]} \sum_{j=1}^{m^{\prime}(y)} \frac{D(y)}{\alpha j},
\end{aligned}
$$

As for the grids in the set $[1, X] /\{\Omega(u) / \Theta\} /\left\{\Omega\left(u^{\prime}\right) / \Theta\right\}$, the number of UAVs covering these grids does not change when the location of UAV $u$ is changed. Thus, we can obtain

$$
\begin{aligned}
& \sum_{x \in[1, X] /\{\Omega(u) / \Theta\} /\left\{\Omega\left(u^{\prime}\right) / \Theta\right\}} \sum_{i=1}^{m(x)} \frac{D(x)}{\alpha i} \\
& =\sum_{y \in[1, X] /\{\Omega(u) / \Theta\} /\left\{\Omega\left(u^{\prime}\right) / \Theta\right\}} \sum_{j=1}^{m^{\prime}(y)} \frac{D(y)}{\alpha j} .
\end{aligned}
$$

Thus, the increment of the potential function is shown as Eq. (17) at the top of the next page.

According to Eq. (14) and (17), we can obtain that $r_{u}$ $r_{u^{\prime}}=\Phi\left(l_{u}, l_{-u}\right)-\Phi\left(l_{u^{\prime}}, l_{-u^{\prime}}\right)$. Therefore, based on the Definition 1, the formulated congestion game is an exact potential game, and it can obtain a stable Nash equilibrium solution.

\section{Simulation Results}

Simulations are conducted to verify the effectiveness of the proposed algorithm. The parameters are shown in TABLE I. A UAV swarm performs data collection task in the jamming environment and $\mathbb{J}$ jammers are randomly distributed in the target area. Since there is no central control UAV, each UAV needs to optimize its position and improve the data collection performance of the entire UAV swarm through information exchange between neighboring UAVs within the communication range $\mathbb{L}$.

Fig. 2(a) show the initial position of the UAV swarm. It can be seen that under the current deployment, the data collection range of some UAVs ( $U_{1}$ and $U_{8}, U_{3}$ and $U_{5}$ ) overlaps, thus the data in the overlapping area will be reduced due to mutual interference, which leads to a low efficiency of data collection. Meanwhile, there are jammers in the collection range of UAVs $U_{1}, U_{3}$ and $U_{8}$, thus the data in these grids with jammers cannot be collected entirely.

Each UAV optimizes its position according to the proposed USDD algorithm, and the optimized results are shown in Fig. 2(b). After the congestion game, each UAV will avoid the grid with jammers and tend to the hot grids with more experiential data. At the same time, each UAV will try to avoid overlapping collection areas, so as to improve the data collection efficiency of the whole swarm.

In the process of congestion game among the UAV swarm, the amount of total data collected is shown in Fig. 3. As the iteration increases, the total amount of data collected by the UAV swarm gradually increases and eventually tends to a stable point. The results obtained are consistent with the analysis in Theorem 1.

Next, we show the impacts of key factors on the performance of the proposed approach in the process of congestion game. In Fig. 4, the initial position of the UAV swarm is generated randomly. When the number of the UAV swarm gradually increases, the total amount of data collected by the UAV swarm gradually increases, but when the number exceeds 16 sorties, the amount of data collected gradually tends to saturation. The reason can be explained that, in this case, the UAV swarm can achieve almost complete coverage to the ground. Additionally, because of the bounded target area and 


$$
\begin{aligned}
\Phi & \left(l_{u}, l_{-u}\right)-\Phi\left(l_{u^{\prime}}, l_{-u^{\prime}}\right) \\
= & \sum_{x \in\{\Omega(u) / \Theta\} \cup\left\{\Omega\left(u^{\prime}\right) / \Theta\right\}} \sum_{i=1}^{m(x)} \frac{D(x)}{\alpha i}-\sum_{y \in\{\Omega(u) / \Theta\} \cup\left\{\Omega\left(u^{\prime}\right) / \Theta\right\}} \sum_{j=1}^{m^{\prime}(y)} \frac{D(y)}{\alpha j} \\
= & \sum_{x \in\{\Omega(u) / \Theta\}} \sum_{i=1}^{m(x)} \frac{D(x)}{\alpha i}+\sum_{x \in\left\{\Omega\left(u^{\prime}\right) / \Theta\right\}} \sum_{i=1}^{m(x)} \frac{D(x)}{\alpha i}-\sum_{y \in\{\Omega(u) / \Theta\}} \sum_{j=1}^{m^{\prime}(y)} \frac{D(y)}{\alpha j}-\sum_{y \in\left\{\Omega\left(u^{\prime}\right) / \Theta\right\}} \sum_{j=1}^{m^{\prime}(y)} \frac{D(y)}{\alpha j} \\
= & \sum_{x \in\{\Omega(u) / \Theta\}}\left(\sum_{i=1}^{m(x)} \frac{D(x)}{\alpha i}-\sum_{i=1}^{m(y)} \frac{D(y)}{\alpha i}-\sum_{j=1}^{m^{\prime}(x)} \frac{D(x)}{\alpha j}\right)+\sum_{y \in\left\{\Omega\left(u^{\prime}\right) / \Theta\right\}} \\
= & \left.\sum_{x \in\{\Omega(u) / \Theta\}}\left(\sum_{i=1}^{m(x)} \frac{D(x)}{\alpha i}-\sum_{i=1}^{m(x)-1} \frac{D(x)}{\alpha i}\right)+\sum_{y \in\left\{\Omega\left(u^{\prime}\right) / \Theta\right\}} \frac{D(y)}{\alpha j}-\sum_{j=1}^{m(y)+1} \frac{D(y)}{\alpha j}\right) \\
= & \sum_{x \in\{\Omega(u) / \Theta\}} \frac{D(x)}{\alpha m(x)}-\sum_{y \in\left\{\Omega\left(u^{\prime}\right) / \Theta\right\}} \frac{D(y)}{\alpha(m(y)+1)} \\
= & \sum_{x \in\{\Omega(u) / \Theta\}} \frac{D(x)}{\alpha m(x)}-\sum_{y \in\left\{\Omega\left(u^{\prime}\right) / \Theta\right\}} \frac{D(y)}{\alpha m^{\prime}(y)} .
\end{aligned}
$$

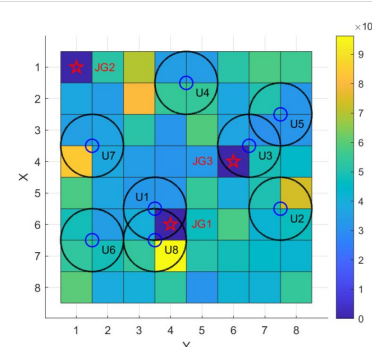

(a)

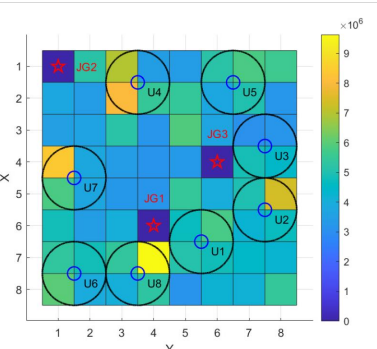

(b)
Fig. 2: (a) Initial deployment of the UAV swarm; (b) The optimized UAV swarm's location. U and JG represent UAV and jammer respectively. The different colors of the grids indicate how much data can be collected.

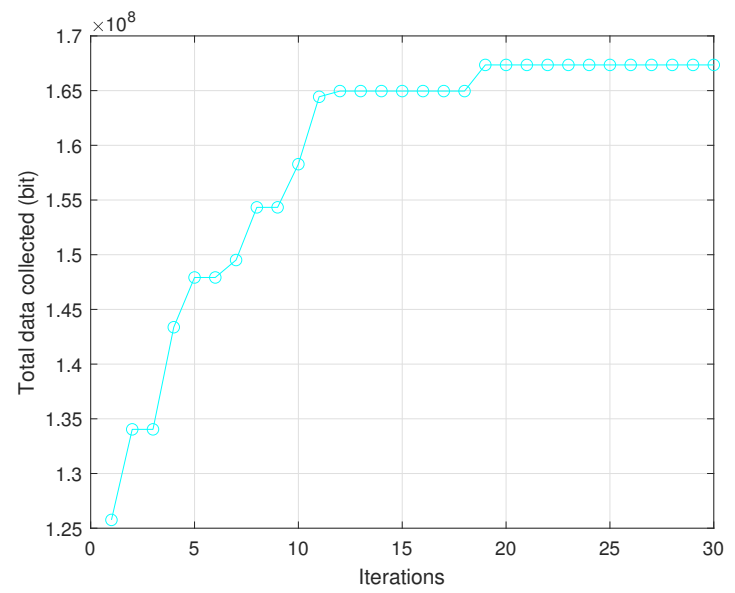

Fig. 3: The amount of collected data by the UAV swarm in the process of the congestion game.
TABLE I: Simulation parameters.

\begin{tabular}{c|c}
\hline \hline Parameters & Value \\
\hline The side length of target area & $8 \times 8 \mathrm{~km}$ \\
\hline The number of grids & $X=64$ \\
\hline The number of jammers & $\mathbb{J}=3$ \\
\hline Average traffic in hot grids & $\lambda^{h} \in[5,10]$ Mbit \\
\hline Average traffic of other grids & $\lambda^{l} \in[3,6] \mathrm{Mbit}$ \\
\hline UAV number & $M=8$ \\
\hline Sensing range of UAV & $L=1 \mathrm{~km}$ \\
\hline Communication range & $\mathbb{L}=2 \mathrm{~km}$ \\
\hline Height of the UAV swarm & $H_{u}=1 \mathrm{~km}$ \\
\hline Communication power & $p_{U} / \sigma^{2}=20 \mathrm{dBW}$ \\
\hline Channel bandwidth & $B=2 \mathrm{MHz}$ \\
\hline Jamming power Path & $p_{J} / \sigma^{2}=23 \mathrm{dBW}$ \\
\hline Coefficient of mutual interference & $\alpha=1$ \\
\hline \hline
\end{tabular}

limited amount of collected data, the average collected data reduces with the increasing of UAV number.

Fig. 5 shows the performance comparison of the proposed algorithm with the benchmark algorithms, including random deployment algorithm, uniform deployment algorithm, and potential game based stochastic optimization algorithm in [14]. As indicated in Fig. 5, the performance of the proposed algorithm is significantly improved compared with the comparing algorithm in [14] and the random deployment scheme. The performance of the proposed algorithm is superior to the uniform deployment algorithm when the number of UAVs is insufficient to complete the full coverage to the target area. When the number of UAVs is more than or equal to 16 sorties, the uniform deployment can achieve slightly higher amount of collected data. This is due to the "selfish" nature of each UAV in the distributed optimization scheme. In the congestion game scenario, each UAV will try to avoid overlapping coverage 


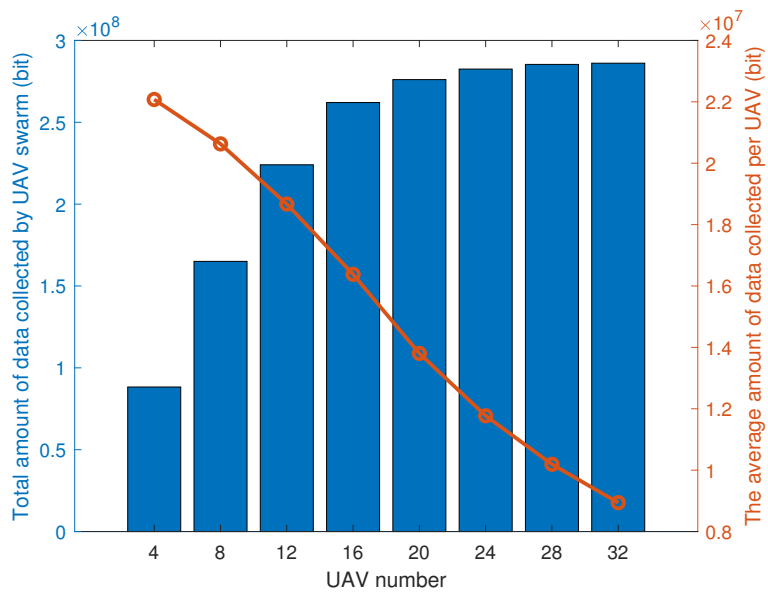

Fig. 4: The impact of the UAV number. The left ordinate represents the change in the total data amount. The right ordinate is the average data amount.

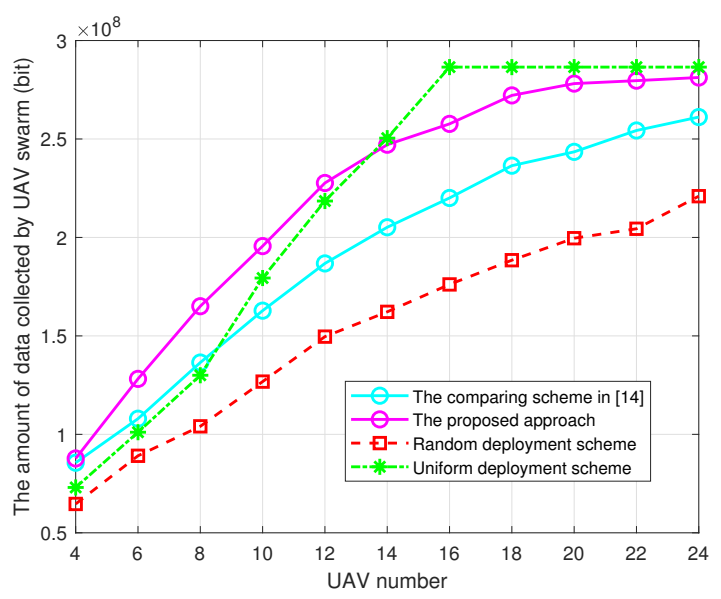

Fig. 5: Performance comparison of different schemes on the total amount of collected data.

with other UAVs. However, all UAVs are comparatively less willing to cover the grids with jamming, so that the performance of distributed optimization is slightly lower than that of the centralized uniform deployment scheme which can achieve full coverage. But, as mentioned earlier, there is resources waste and performance loss when the UAV swarm is insufficient to achieve complete coverage. Additionally, it will face serious threat of malicious jamming. The comparison results between the proposed distributed algorithm and the uniform deployment algorithm are shown in Fig. 6 when the $\mathrm{UAV}$ is deployed for 16 sorties.

\section{CONCLUSION}

This paper investigated the distributed deployment problem for a UAV swarm in the hostile environments. A congestion game was formulated to model the interaction among the UAV swarm, and a distributed deployment algorithm was proposed to achieve flexible and autonomous deployment optimization, and enhance anti-jamming capability. The Nash equilibrium of the formulated congestion game was analyzed, and the

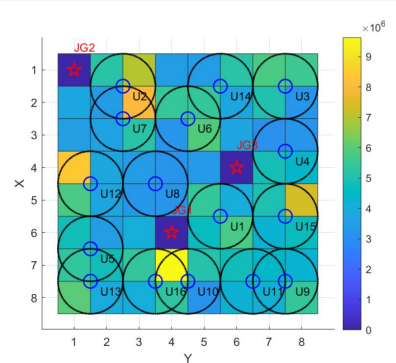

(a)

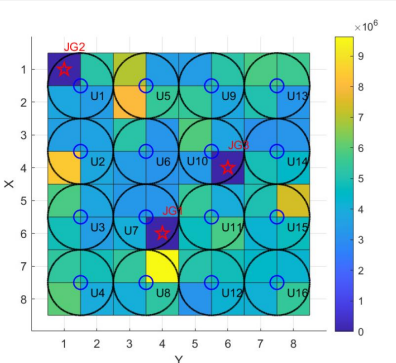

(b)
Fig. 6: (a) Distributed deployment result by the proposed approach; (b) Uniform deployment result.

simulation results indicated that the proposed method had better performance compared with the benchmark algorithms.

\section{REFERENCES}

[1] M. Zhang, S. Fu, and Q. Fan, "Joint 3d deployment and power allocation for uav-bs: A deep reinforcement learning approach," IEEE Wireless Commun. Lett., pp. 1-1, 2021.

[2] S.-C. Noh, H.-B. Jeon, and C.-B. Chae, "Energy-efficient deployment of multiple uavs using ellipse clustering to establish base stations," IEEE Wireless Commun. Lett., vol. 9, no. 8, pp. 1155-1159, 2020.

[3] A. Fotouhi, H. Qiang, M. Ding, M. Hassan, L. G. Giordano, A. GarciaRodriguez, and J. Yuan, "Survey on uav cellular communications: Practical aspects, standardization advancements, regulation, and security challenges," IEEE Commun. Surv. Tutor., vol. 21, no. 4, pp. 3417-3442, 2019.

[4] Y. Wang, Z.-Y. Ru, K. Wang, and P.-Q. Huang, "Joint deployment and task scheduling optimization for large-scale mobile users in multi-uavenabled mobile edge computing," IEEE Trans. on Cybern., vol. 50, no. 9 , pp. 3984-3997, 2020.

[5] S. Sun, G. Zhang, H. Mei, K. Wang, and K. Yang, "Optimizing multiuav deployment in 3-d space to minimize task completion time in uavenabled mobile edge computing systems," IEEE Commun. Lett., vol. 25, no. 2, pp. 579-583, 2021.

[6] M. Mozaffari, W. Saad, M. Bennis, and M. Debbah, "Efficient deployment of multiple unmanned aerial vehicles for optimal wireless coverage," IEEE Commun. Lett., vol. 20, no. 8, pp. 1647-1650, 2016.

[7] Y.-C. Kuo, J.-H. Chiu, J.-P. Sheu, and Y.-W. P. Hong, "Uav deployment and iot device association for energy-efficient data-gathering in fixedwing multi-uav networks," IEEE Trans. Green Commun. Netw., pp. 1-1, 2021.

[8] J. Wang, M. Liu, J. Sun, G. Gui, H. Gacanin, H. Sari, and F. Adachi, "Multiple unmanned-aerial-vehicles deployment and user pairing for nonorthogonal multiple access schemes," IEEE Internet Things J., vol. 8, no. 3, pp. 1883-1895, 2021.

[9] X. Zhang and L. Duan, "Energy-saving deployment algorithms of uav swarm for sustainable wireless coverage," IEEE Trans. Veh. Technol., vol. 69 , no. 9, pp. 10320-10335, 2020.

[10] E. Koyuncu, M. Shabanighazikelayeh, and H. Seferoglu, "Deployment and trajectory optimization of uavs: A quantization theory approach," IEEE Trans. Wireless Commun., vol. 17, no. 12, pp. 8531-8546, 2018.

[11] M. López-Benítez, C. Majumdar, and S. N. Merchant, "Aggregated traffic models for real-world data in the internet of things," IEEE Wireless Commun. Lett., vol. 9, no. 7, pp. 1046-1050, 2020.

[12] C. Han, A. Liu, H. Wang, L. Huo, and X. Liang, "Dynamic anti-jamming coalition for satellite-enabled army iot: A distributed game approach," IEEE Internet Things J., vol. 7, no. 11, pp. 10932-10944, Nov. 2020.

[13] D. Liu, J. Wang, K. Xu, Y. Xu, Y. Yang, Y. Xu, Q. Wu, and A. Anpalagan, "Task-driven relay assignment in distributed uav communication networks," IEEE Trans. Veh. Technol., vol. 68, no. 11, pp. 11 003-11 017, 2019.

[14] H. Dai, H. Zhang, C. Li, and B. Wang, "Efficient deployment of multiple uavs for iot communication in dynamic environment," China Communications, vol. 17, no. 1, pp. 89-103, 2020. 